\title{
La critique de l'épistémologie classique et ses implications pédagogiques chez John Dewey et Karl Popper
}

The critique of classical epistemology and its educational implications in J.

Dewey and K. Popper

\section{Alain Firode}

\section{(2) OpenEdition}

\section{Journals}

Édition électronique

URL : https://journals.openedition.org/ree/1943

DOI : $10.4000 /$ ree.1943

ISSN : 1954-3077

Éditeur

Nantes Université

Référence électronique

Alain Firode, "La critique de l'épistémologie classique et ses implications pédagogiques chez John Dewey et Karl Popper », Recherches en éducation [En ligne], 34 | 2018, mis en ligne le 01 novembre

2018, consulté le 09 mars 2022. URL : http://journals.openedition.org/ree/1943 ; DOI : https://doi.org/ $10.4000 /$ ree. 1943

\section{(c) (†) $\ominus$}

Recherches en éducation est mise à disposition selon les termes de la Licence Creative Commons Attribution - Pas d'Utilisation Commerciale - Pas de Modification 4.0 International. 


\title{
La critique de l'épistémologie classique et ses implications pédagogiques chez John Dewey et Karl Popper
}

\begin{abstract}
Alain Firode ${ }^{1}$
Résumé

John Dewey et Karl Popper ont en commun d'avoir critiqué les théories classiques de la connaissance en leur opposant une épistémologie naturaliste et évolutionniste dans laquelle le connaître est pensé comme un prolongement de l'activité vitale. Quoiqu'ils visent la même cible, les deux auteurs ne lui adressent pas le même reproche. Le tort des philosophes classiques, pour Dewey, est d'avoir accepté sans recul critique "l'épistémologie du spectateur " qui identifie la connaissance à une représentation adéquate du réel ; selon Popper, il est d'être restés prisonniers de "l'épistémologie subjectiviste " qui limite par principe la connaissance à ce qui est su d'un sujet. De ces deux façons d'envisager l'erreur fondamentale de la philosophie occidentale découlent deux critiques différentes, et par certains côtés opposées, de la pédagogie traditionnelle.
\end{abstract}

Si l'existence de points communs entre l'épistémologie poppérienne et le pragmatisme nordaméricain n'a pas échappé aux historiens de la philosophie, les études qui ont abordé cette question paraissent s'être surtout concentrées sur les relations entre Karl Popper et Charles Sanders Peirce (Chauviré, 2003). Les rapports entre Popper et cet autre représentant majeur du pragmatisme qu'est John Dewey ne semblent pas en revanche avoir suscité autant d'intérêt. Les points de convergences entre les deux auteurs, pourtant, sont nombreux et frappants : Popper et Dewey ont eu l'un et l'autre pour ambition de naturaliser l'épistémologie en inscrivant l'étude de la connaissance scientifique dans une perspective évolutionniste d'inspiration darwinienne ; ils ont rejeté avec la même fermeté la théorie traditionnelle de la science comme connaissance certaine (épistémè), théorie à laquelle ils ont l'un et l'autre opposé une épistémologie analysant la connaissance à la façon d'un processus naturel de résolution de problème (« l'enquête » chez Dewey, le processus de «conjectures et réfutations » chez Popper). Enfin, si les préoccupations éducatives découlant de ces analyses épistémologiques sont évidemment beaucoup plus manifestes dans l'œuvre de Dewey, elles n'en sont pas pour autant absentes chez Popper, comme en témoignent ses écrits de jeunesse, pour l'essentiel consacrés à des questions d'ordre pédagogique (Popper, 2006), ainsi que la présence dans l'œuvre de la maturité de nombreux passages concernant la théorie de l'apprentissage et le développement psychologique de l'enfant.

C'est cette proximité de vues qui, nous semble-t-il, rend intéressante la confrontation des deux auteurs, en ce qu'elle permet d'interroger la pensée du philosophe américain depuis un site inhabituel, susceptible, nous l'espérons, de faire naître un dialogue critique inédit et fécond. La philosophie de Dewey, il est à peine besoin de le rappeler, constitue l'une des sources majeures du progressisme pédagogique contemporain, ce qui en fait bien évidemment l'une des cibles favorites de ses adversaires conservateurs. Comme l'a justement souligné Michel Fabre (2016) toutefois, ces critiques, qui se bornent le plus souvent à reprendre les arguments avancés dans le célèbre texte d'Hannah Arendt à l'encontre des idées progressistes dans l'école américaine des années 1960 (Arendt, 1972), manquent leur cible, dans la mesure où Dewey lui-même (Dewey, 2011) n'avait pas manqué d'apercevoir et de critiquer les dérives auxquelles la mise en pratique de ses propres idées avait pu donner lieu. De là l'intention de cette étude qui est de contribuer à renouveler les termes du débat concernant le progressisme éducatif en confrontant la pensée du philosophe américain à celle d'un auteur qui, tout en étant proche de lui (par son

\footnotetext{
${ }^{1}$ Professeur des universités, Laboratoire «Recherches en Éducation, Compétences, Interactions, Formation, Éthique, Savoirs » (RECIFES), Université d'Artois.
} 
attitude critique à l'égard des épistémologies classiques et des pratiques d'enseignement dont elles constituent le fondement), aboutit cependant à de tout autres conclusions, aussi bien en ce qui concerne la question épistémologique (§ I) que la question pédagogique (§ II). L'objectif, autrement dit, est de montrer que le progressisme éducatif contemporain, dont Dewey a explicité les fondements avec la plus grande cohérence logique, peut être questionné sur des bases théoriques différentes de celles dont usent ordinairement les critiques d'inspiration conservatrice, en tenant donc à l'écart les questions autour desquelles, habituellement, tourne la discussion (la crise de l'autorité, la place du maître, la liberté des enfants, etc.).

\section{La critique de l'épistémologie classique}

\section{- Deux façons d'envisager la naturalisation de l'épistémologie}

Popper et Dewey ont en commun de s'être appuyés sur une approche naturaliste et évolutionniste de la connaissance, d'inspiration darwinienne, pour aborder le problème épistémologique. La connaissance, chez les deux auteurs, apparaît comme un prolongement de l'activité vitale : de même que l'animal cherche à rétablir son équilibre avec le milieu lorsque celui-ci est troublé par un phénomène inattendu, de même le scientifique (et plus généralement le sujet connaissant) cherchent à recomposer, en inventant de nouvelles théories, la stabilité intellectuelle que procurait l'ancienne théorie avant d'être réfutée. La connaissance sous toutes ses formes, autrement dit, consiste en un processus de "problem solving", la résolution de problème étant pour les deux auteurs l'activité primitive de l'être vivant (Popper, 1997), le moteur du processus "continuel de déséquilibres et de restaurations d'équilibre » que constitue la vie elle-même (Dewey, 1993, p.85). Les deux philosophes s'accordent donc sur le fait qu'on ne pense pas «gratuitement » mais pour répondre à une perturbation introduite dans une situation auparavant considérée comme stable, bref pour répondre à un problème. Sur ce point la théorie de "l'enquête » chez Dewey ne s'écarte guère des analyses de Popper concernant le processus de «conjectures et réfutations" (Popper, 1985) auquel obéit selon lui tout effort de connaissance : "Tout ce qu'on peut dénommer "connaissance" ou "objet connu" désigne une question à laquelle une réponse a été apportée, une difficulté surmontée, la dissipation d'une confusion, une incohérence muée en cohérence, une perplexité maîtrisée » (Dewey, 2008, p.243).

Chez Dewey, cette approche naturaliste et évolutionniste de l'épistémologie conduit à l'abandon de la conception traditionnelle de la science comme "quête de la vérité ", entendue au sens d'un effort pour représenter fidèlement une réalité préexistante. Même lorsqu'elle fait l'objet d'une élaboration hautement abstraite et intellectuelle, la connaissance reste toujours pour le philosophe américain un processus d'adaptation (d'équilibration avec le milieu), ayant pour fin « la transformation contrôlée ou dirigée d'une situation indéterminée en une situation qui est si déterminée en ses distinctions et relations constitutives qu'elle convertit les éléments de la situation originelle en un tout unifié »(Dewey, 1993, p.169). Ainsi, selon Dewey, toute la fécondité de la révolution expérimentale accomplie au $\mathrm{XVII}{ }^{\mathrm{e}}$ siècle provient-elle de ce que les savants qui l'ont portée ont su renouer, par-delà l'héritage intellectualiste de la culture antique et médiévale, avec l'idée que la connaissance n'est pas un moyen d'accès au réel, mais une modalité d'échange avec le milieu : non pas la représentation d'une réalité antécédente, supposée exister en soi, mais le résultat d'un processus actif (l'expérimentation) par lequel l'environnement, transformé en un complexe de relations, est rendu disponible pour l'action humaine. Telle est à ses yeux la signification épistémologique de la physique mathématique dans son ensemble : en déterminant les propriétés des corps de façon quantitative et relationnelle, et non plus qualitative, la science moderne nous fait passer d'un monde de choses fixes (de substances) à un monde d'événements interconnectés, sur lesquels nous pouvons agir (Dewey, 2008, p.145 sq.)

II en va tout autrement chez Popper. L'épistémologie poppérienne, en effet, établit certes une filiation entre l'activité vitale et l'activité intellectuelle, mais non comme chez Dewey une réelle continuité. Quoique la pensée scientifique soit un effort pour faire cesser une difficulté, celle-ci 
pour Popper n'est cependant pas identique au trouble vécu que subissent l'animal ou le sujet engagés dans l'action lorsqu'ils se trouvent confrontés à l'imprévu, de même que l'équilibre qu'il s'agit alors de rétablir ne consiste pas dans cet état mental stable qu'est la croyance. À plusieurs reprises le philosophe insiste sur les raisons qui rendent à ses yeux impossible une telle assimilation. La première tient au rapport à l'erreur : alors qu'une conduite adaptative cherche par nature à éviter l'erreur, la recherche scientifique requiert à l'inverse une attitude qui va audevant de l'erreur pour l'éliminer. Telle est la différence entre l'amibe et Einstein : l'amibe (ou le croyant) « détestent » l'erreur, alors que l'erreur intéresse le savant (Popper, 1991, p.133). Bref, Popper tient pour impensable l'audace constitutive de la pensée scientifique dans l'hypothèse où la science serait une modalité de l'adaptation. La seconde raison réside dans l'impossibilité d'assimiler les connaissances scientifiques à des instruments permettant, comme les croyances, d'anticiper les effets de nos actions. Si les théories scientifiques étaient des instruments, en effet, celles-ci devraient être testées à la manière dont on teste un outil (par exemple, un fuselage d'avion dans une soufflerie) : non pour les réfuter, les invalider définitivement, mais pour en marquer les limites d'utilisation. Or ce n'est pas ainsi que procèdent les savants qui, au contraire, n'hésitent pas à abandonner une fois pour toutes les théories ayant rencontré le démenti du réel (Popper, 1990, p.133-136). Si les scientifiques font ce qu'ils font, c'est donc selon Popper qu'ils tiennent les théories pour autre chose que pour de simples instruments énonçant « des formules en vue de la prédiction de la probabilité d'un événement observable » (Dewey, 2008, p.222). À savoir pour des conjectures visant à cerner la structure des mondes possibles dont notre monde fait partie. Considérées d'un point de vue logique, en effet, les lois physiques selon Popper ne sont pas des énoncés affirmatifs formulant des prévisions conditionnelles (du type : "si l'on fait ceci, alors il s'en suivra tel effet »), mais des énoncés négatifs proscrivant l'apparition de certains événements. Aussi l'objectif des savants, selon Popper, est-il de définir des univers possibles par l'interdiction d'y voir se produire certains phénomènes (par exemple, affirmer la loi de conservation de l'énergie revient, d'un point de vue logique, à nier l'existence du mouvement perpétuel et non à prédire l'apparition conditionnelle de tel ou tel événement).

Pour expliquer ces écarts entre la conduite adaptative et la recherche scientifique, l'épistémologie poppérienne, à la différence des épistémologies classiques, ne suppose pas le sujet humain doté d'une quelconque faculté métaphysique transcendante lui permettant d'échapper au monde naturel. Ceux-ci sont rendus possibles, selon Popper, en raison d'une propriété tout à fait naturelle du langage humain, quoiqu'inconnue du langage animal, qui est sa capacité à désincorporer les états mentaux du sujet, à les convertir en connaissances " objectives », détachées de leur porteur organique car déposées dans un support extrasomatique. Si les savants peuvent inverser le rapport naturel à l'erreur, ou encore s'ils peuvent considérer comme définitivement invalidées les conjectures ayant rencontré le désaveu du réel (bref, s'ils peuvent faire ce qu'ils font), c'est qu'ils se sont désengagés de leurs croyances en les formulant : c'est, comme dit Popper (1991, p.369), qu'ils envoient «leurs théories mourir à leur place ". D'où il suit que tout effort de connaissance, dans l'épistémologie poppérienne, requiert un sujet "désimpliqué ", désengagé de la situation, dont l'intérêt et l'attention se sont reportés sur des objets intellectuels (théories, concepts) détachés de tout enjeu vital (personnel ou social) parce que rendus autonomes par le pouvoir objectivant du langage. Sur ce point, l'opposition avec Dewey est bien évidemment frontale. La pensée poppérienne, considérée d'un point de vue pragmatiste, ne peut apparaître que comme un avatar de cette "épistémologie du spectateur » que le philosophe américain n'a cessé de combattre au cours de sa carrière. Penser la connaissance dans une perspective évolutionniste, pour Dewey, revient nécessairement à abandonner l'idée d'un sujet situé devant un monde qui se donne à connaître pour concevoir au contraire la connaissance comme un processus immanent à la réalité naturelle, une interaction adaptative entre un organisme situé dans un univers qui l'inclut et l'implique nécessairement, et son milieu, physique et social ("le connaître est quelque chose qui se produit au sein de la nature »- Dewey, 2008, p.261). Non pas «l'acte d'un spectateur se tenant en dehors de la scène naturelle et sociale, mais l'acte d'un participant» (p.212). Les conséquences pédagogiques de cette opposition, sur lesquelles nous reviendrons bientôt, ne sont pas moins radicales. Pour Dewey, il importe au plus haut point à la formation intellectuelle de l'élève que celui-ci se sente personnellement impliqué dans toutes les activités cognitives qui lui sont proposées. Du point de vue poppérien, au contraire, cet esprit de sérieux qui parcourt toute la 
pédagogie de Dewey, ce souci de constamment relier la connaissance à des « enjeux » réels et sociaux dans lesquels le sujet se trouve "pris ", ferment infailliblement l'accès à la pensée scientifique, laquelle requiert au contraire, pour les raisons qui viennent d'être mises en évidence, un désengagement personnel du sujet.

\section{- Deux critiques des « doctrines de la certitude»}

Chez les deux auteurs, autre point commun remarquable, l'assimilation de la connaissance à une activité de résolution de problème entraine une critique radicale de l'idée de certitude. En tant qu'elle est l'aboutissement d'un processus visant à résoudre un problème, toute connaissance est par nature provisoire, l'équilibre qu'elle procure étant nécessairement appelé à être rompu par l'apparition de nouveaux problèmes suscités par la solution elle-même. II n'est donc aucune connaissance certaine parce qu'immédiate (telle la certitude sensible des empiristes ou l'évidence des cartésiens) ni non plus aucune connaissance certaine parce qu'établie à la suite d'une inférence (Dewey, 1993, chap. VIII, p.209 sq.). L'enquête (chez Dewey) ou le processus de «conjectures et réfutations » (chez Popper) ne peuvent pas plus aboutir à une solution définitive qu'ils ne peuvent se fonder sur une vérité de départ indiscutable, sensible ou intellectuelle. Ainsi, I'hypothèse ayant fait l'objet d'une « préférence critique » pour la solution d'un problème, selon Popper, reste-t-elle une hypothèse: comme telle, elle est par nature provisoire et rien ne s'oppose à ce qu'elle soit un jour ou l'autre remise en question. De même pour le philosophe américain : "les résultats antérieurs (qui) sont pris et utilisés sans réexamen 》 pour la nécessité d'une enquête « ne sont pas délivrés de la nécessité d'être réexaminés et reconstruits » en cas de besoin (Dewey, 1993, p.210-211). La croyance à l'existence de vérités indiscutables, pour Popper comme pour Dewey, n'est jamais qu'un préjugé de nature dogmatique nuisible au progrès scientifique (p.212), une illusion devenue intenable à la suite des avancées de la science contemporaine (particulièrement de la Théorie de la Relativité d'Einstein, également invoquée en ce sens par les deux auteurs).

Cette critique de la croyance dans l'existence de connaissances certaines se double, chez Dewey comme chez Popper, d'une enquête sur les origines de l'idéal épistémique de certitude. Comment expliquer que la science ait été assimilée, à tort, avec une « quête de la certitude »? Pour répondre à cette question Dewey se livre, dans La quête de la certitude, à un examen généalogique du besoin de certitude. Dans la perspective darwinienne qui sous-tend ses analyses, le moteur primitif de l'activité humaine ne peut qu'être d'ordre biologique : il réside dans la recherche de la sûreté, autrement dit dans le besoin de se prémunir contre les aléas de l'environnement (Dewey, 2008, p.23). L'idée d'une connaissance "désintéressée » en quête de " certitude ", quant à elle, n'est jamais qu'une fiction qui ne résiste pas au dévoilement de ses origines : celle-ci a sa source dans la pensée grecque classique, à une époque où la faiblesse des moyens techniques permettant de contrôler les phénomènes naturels a conduit à inventer et à promouvoir l'idée d'un rapport contemplatif au monde (la « théoria ») d'où l'incertitude et donc le risque corrélatif de toute action se trouveraient exclus par principe (p.58). Bref, selon Dewey c'est pour échapper illusoirement aux vicissitudes du réel que la pensée grecque et toute la tradition philosophique occidentale qu'elle a inspirée ont créé et exalté le mythe d'une connaissance purement contemplative en même temps qu'elles se sont efforcées de dévaluer intellectuellement et socialement (cf. l'esclavage antique) l'activité du sujet réel, exposé et fragile car engagé dans le monde (Dewey, 2008, p.111 sq.)

La critique poppérienne de l'idéal de certitude, quant à elle, est d'une toute autre nature. Selon Popper, en effet, l'identification de la recherche scientifique à une quête de la certitude n'est pas la conséquence d'un quelconque préjugé intellectualiste hérité de la philosophie grecque, mais la suite inévitable de la conception "subjectiviste" de la connaissance que la plupart des épistémologies ont emprunté sans recul critique au sens commun. Leur erreur commune - en quoi réside leur « subjectivisme " - consiste à ne pas distinguer la connaissance objective de la connaissance subjective, la connaissance comme produit de l'esprit humain, implantée dans des supports extra-mentaux (livres, revues, etc.), de la connaissance comme état mental, implantée dans la conscience du sujet. Ainsi, c'est parce qu'elle méconnait la nature objective (au sens qui vient d'être indiqué, où " objectif » signifie « extra-mental ») de la connaissance scientifique, que 
toute l'épistémologie classique, selon Popper, assimile à tort celle-ci avec cet état psychologique qu'est la certitude. Une fois dissipé ce préjugé "subjectiviste", il devient clair que la connaissance scientifique, en tant que " connaissance objective ", connaissance " sans sujet connaissant » (Popper, 1991, p.181), n'a rien à voir avec une quelconque «quête de la certitude ". La validation des énoncés scientifiques, selon ce point de vue, dépend uniquement de leurs propriétés logiques objectives, lesquelles sont indépendantes des états mentaux, tels que le doute ou la certitude, éprouvés par le savant. Ainsi, l'impossibilité où se trouve la science de parvenir à établir des connaissances certaines n'est-elle l'indice d'aucun défaut logique : la certitude est non seulement une fin que la connaissance scientifique ne peut atteindre, c'est aussi une fin vers laquelle elle n'a pas à tendre. En tant que connaissance " objective » (c'est-àdire indépendante du sujet connaissant), une connaissance scientifique peut être absolument satisfaisante tout en étant « incertaine ». C'est même là, pour Popper, une nécessité : en effet, la possibilité pour une théorie scientifique d'être réfutée (donc son incertitude) est la marque qu'elle atteint effectivement la réalité «en soi » (les réfutations désignant les « points de contact »Popper, 1985, p.178 - de la théorie avec le monde) en sorte qu'une connaissance irréfutable et certaine, si elle venait à exister, serait aussi une connaissance dépourvue de tout rapport avec le réel, une connaissance sans « contenu de vérité » (Popper, 1991, p.305).

L'abandon de l'idéal de certitude, dans l'épistémologie poppérienne, n'implique donc nullement de renoncer à l'idée que la connaissance scientifique consiste en une "quête de la vérité » (au sens réaliste du terme), l'incertitude de la science étant justement ce qui garantit l'existence de son rapport au réel. II implique, en revanche, que la science soit rigoureusement pensée comme un produit objectif et non comme un état de l'esprit humain. Ainsi, à la différence de «l'enquête » pragmatiste, la Forschung poppérienne ne commence-t-elle pas par le « doute » et ne s'achève pas davantage par la "fixation de la croyance » dans l'esprit du sujet. Son terminus a quo est la " critique » et son terminus ad quem l'établissement d'une "préférence critique », étant entendu que la critique est indépendante du doute (" on peut critiquer sans douter », Popper, 1991, p.226) et que l'établissement d'une préférence critique n'implique rien quant aux croyances personnelles du sujet qui la formule.

Ces divergences, une fois encore, ne vont pas sans conséquences pédagogiques, au moins en ce qui concerne le domaine de l'enseignement scientifique. L'un des soucis les plus constants de la didactique actuelle des sciences est en effet de développer des méthodes d'enseignement qui concilient deux exigences : d'une part celle de ne pas présenter la science aux élèves comme un corpus de vérités fixes et de certitudes intangibles mais comme un processus "qui évolue en permanence, remettant en cause les vérités d'hier » (Johsua \& Dupin, 2003, p.33) ; d'autre part celle de leur faire néanmoins clairement saisir la valeur des connaissances scientifiques, ce par quoi elles s'élèvent au-dessus de la connaissance commune (p.59). II s'agit, autrement dit, d'éviter tout à la fois le « dogmatisme » positiviste (p.33) et le « relativisme » sceptique (p.59). La solution de ce problème, chez les auteurs se réclamant actuellement du " constructivisme » et du "socioconstructivisme », fait appel à des idées plus ou moins proches de celles défendues par Dewey : elle consiste à prôner des procédés didactiques qui fassent apparaître les concepts et les théories scientifiques comme des représentations mentales par lesquelles les sujets organisent collectivement leur expérience afin de lui conférer une signification humaine et sociale universellement partageable (cf. Le Moigne qui souligne à juste titre la convergence, sur ce point, entre les épistémologies «constructiviste » et «pragmatiste », 1999, p.55 et p.68). Dans les savoirs scientifiques, les professeurs et leurs élèves sont ainsi invités à voir des « représentations standardisées et fiables » (Fourez, 2003, p.22) « construites collectivement », dont l'un des principaux avantages est de "favoriser la communication » intersubjective et l'action collective (p.17). Détachée de son rapport à la notion de "vérité objective " (tenue par certains auteurs constructivistes pour aussi suspecte que celle de "vérité révélée ", Le Moigne, 1999, p.68), la valeur des connaissances scientifiques se mesure alors « en fonction des projets qu'elles rendent possibles » (Fourez, 2003, p.22), en tant qu'« elles visent à éclairer des situations en vue d'en parler ou d'agir » (ibid. $)^{2}$.

\footnotetext{
${ }^{2}$ Cette tendance à faire résider la valeur de la science ailleurs que dans son rapport à la vérité se retrouve dans de nombreux ouvrages contemporains consacrés à l'enseignement scientifique. Ainsi, pour André Giordan (2016), une théorie ne doit-elle pas être estimée en fonction de sa correspondance au réel : elle n'est "ni "conforme" ni "inadéquate". Elle est seulement
} 
Quoique cette façon de présenter la science permette effectivement d'éviter les deux écueils opposés que sont le relativisme et le positivisme, le détour par l'épistémologie poppérienne fait voir qu'elle ne constitue toutefois pas la seule façon possible d'envisager les conditions d'un enseignement scientifique non dogmatique. Selon Popper, on l'a vu, la rupture avec le dogmatisme n'exige pas l'abandon des prétentions de la science à valoir comme connaissance vraie de la réalité en soi, mais l'abandon de sa prétention à valoir comme connaissance sue du sujet. D'où il suit que les conditions d'un enseignement critique des sciences, si elles venaient à être développées dans un sens conforme aux thèses poppériennes, passeraient par la mise en place de procédés pédagogiques tout autres que ceux recommandés par les tenants du progressisme. Des procédés qui, à l'inverse de ce que prône Dewey, viseraient à faire apparaître l'apprentissage d'une science comme la découverte d'un édifice logique extérieur au sujet humain et indifférent à ses croyances personnelles (une " cathédrale » en constante évolution pour reprendre l'image poppérienne, Popper, 1991, p.201 et p.287). Les deux pensées, une fois encore, conduisent à des conclusions diamétralement opposées.

\section{La critique de la pédagogie traditionnelle}

\section{- La continuité des apprentissages scolaires et des apprentissages informels (Dewey)}

L'ensemble des réflexions pédagogiques de Dewey peut être vue comme une tentative pour mettre l'école et la société en conformité avec la conception non métaphysique de la connaissance qu'appellent inévitablement, selon lui, les bouleversements épistémologiques suscités par le progrès scientifique à l'âge moderne. Qu'implique une telle mise en adéquation?

Écartons, en premier lieu, la réponse qui pourrait se tirer d'une lecture superficielle des écrits pédagogiques du philosophe américain. Contrairement à ce qu'affirment fréquemment les détracteurs du pragmatisme, en effet, les réformes prônées par l'auteur de Démocratie et éducation ne débouchent pas sur l'idée que le sens de tout apprentissage devrait se réduire à sa fonction "utilitaire » et immédiatement pratique. II est tout à fait légitime selon Dewey qu'un chercheur ou qu'un élève s'intéressent, par exemple, aux mathématiques ou à la physique indépendamment de toute considération pratique immédiate. L'erreur est de prendre ce désintérêt vis-à-vis des applications immédiates et particulières de tel ou tel savoir pour un désintérêt vis-à-vis de toute application pratique en général et d'inventer ainsi le mythe d'un intérêt naturel et gratuit pour la "Vérité », lequel serait d'une nature supérieure à toute préoccupation pratique et sociale (Dewey, 2008, p.171). II importe, par ailleurs, de ne pas avoir une conception étroitement instrumentaliste de ce qui est qualifié d'«utile » par les auteurs se réclamant du pragmatisme. Selon Dewey une activité peut être à bon droit qualifiée "d'utile " dans un sens qui déborde la catégorie de ce qui "sert » à titre d'instrument technique dès lors que cette « utilité » réside simplement dans l'élargissement de l'expérience personnelle de celui qui la pratique. Ainsi serait-il absurde, note à ce sujet Dewey, de considérer qu'un enseignement n'a une «valeur qui en justifie l'étude " qu'à la condition "que l'élève ou le maître puisse montrer à quoi il pourra servir à un moment donné du futur » (Dewey, 2011, p.332). La théorie pragmatiste de l'éducation, dans la version qu'en propose Dewey, débouche sur une forme d'humanisme pédagogique qui fait résider les sens des apprentissages dans l'approfondissement de l'expérience personnelle du sujet - dans une intensification de sa vie - et nullement sur une forme d'utilitarisme techniciste.

La critique adressée à la pédagogie traditionnelle ne vise donc pas la légitimité des enseignements "abstraits". La cible véritable réside ailleurs, dans l'idée que les objets intellectuels constitutifs de ces savoirs «théoriques " formeraient un «monde » doué d'une existence et d'une signification autonomes, indépendantes de leur relation à l'expérience humaine et sociale. Ainsi Dewey $(2008$, p.181) s'en prend-il aux théories qui, telle la théorie

“opératoire" on inefficiente » (p.97). L'explication scientifique, dans ces conditions, n'est pas une tentative pour approcher la réalité sous-jacente aux phénomènes mais un moyen de « donner du sens à une situation » (p.156). 
platonicienne des idées, provoquent « une hypostase des objets de pensée, en les situant dans un domaine à part, celui de l'Étre transcendant ». L'éducation, selon le philosophe américain, devient « lointaine et morte, abstraite et livresque » toutes les fois que le savoir est identifié à ce qui se trouve "emmagasiné dans des symboles difficilement traduisibles en actes et objets familiers » (Dewey, 2011, p.87), comme si les objets intellectuels existaient « dans un monde en soi » (ibid.). Dès lors, « les liens qui unissent le contenu des études scolaires aux habitudes et aux idéaux du groupe social sont déguisés et dissimulés. Ils deviennent si lâches qu'il semble parfois qu'il n'y en a pas, comme si le contenu pouvait exister simplement en tant que connaissance à son profit exclusif et comme si l'étude était simplement l'acte de le maîtriser pour lui-même, indépendamment de toute valeur sociale»(p.268). De là l'objectif principal de la pédagogie progressiste qui sera justement de «mettre en lumière ce lien que l'on perd si souvent et de montrer avec quelques détails que les éléments constitutifs essentiels du cours des études ont un contenu social et une fonction sociale » (p.268-269). II importe pour cela, du point de vue des méthodes pédagogiques, que les apprentissages scolaires ne se distinguent pas structurellement des apprentissages extra-scolaires informels : «En effet, lorsque les écoles abandonnent les conditions éducatives qui ont cours dans l'environnement extra-scolaire, elles substituent nécessairement un esprit livresque et pseudo-intellectuel à un esprit social » (p.119). Ainsi l'école devra-t-elle s'efforcer de « reproduire les situations de la vie » (p.247) afin de donner aux élèves l'occasion "d'acquérir et de mettre à l'épreuve les idées et le savoir dans des occupations reproduisant effectivement des situations sociales importantes » (ibid.).

\section{- Une critique non progressiste de la pédagogie traditionnelle}

(Popper)

Quoique Popper, à la différence de Dewey, n'ait pas cherché à développer en détail les implications éducatives de son épistémologie, celles-ci peuvent néanmoins être reconstituées sans trop de risque d'égarement. II est clair, en effet, que les analyses poppériennes ne débouchent en rien sur la thèse progressiste selon laquelle la place des savoirs dans les programmes scolaires devrait se justifier par leur rôle social et leur portée, sinon utilitaire, du moins fonctionnelle. En tant qu'elles sont dépositaires d'un «contenu objectif de vérité » les connaissances déposées dans les livres, selon Popper, sont intrinsèquement précieuses, indépendamment de la signification qu'elles présentent pour le sujet et la société. On comprend également, dans ces conditions, que leur transmission, telle qu'elle a lieu dans l'école, ne soit pas réductible à l'appropriation d'outils destinés à élargir notre expérience ou à promouvoir la vie interhumaine : elle consiste fondamentalement dans la découverte d'un monde, d'un univers réel clos sur lui-même, supposé préexister à l'acte par lequel le sujet l'appréhende. Considérée d'un point de vue poppérien, c'est cette substantialité des savoirs qui explique et légitime en dernière analyse l'existence même de l'école, le propre des savoirs « objectifs » étant précisément de ne pouvoir être transmis par simple participation à la vie d'un groupe humain, par l'accomplissement d'activités coopératives suscitant des interactions avec un environnement à la fois physique et social. L'école, ainsi comprise, est au contraire l'institution qui nous apprend à nous rapporter aux savoirs comme à des objets réels possédant une existence autonome. Sa vocation profonde, si l'on suit les analyses que Popper (1985, p.206 sq.) consacre à l'enseignement des premiers physiciens de la Grèce archaïque, est d'instituer chez l'élève ce rapport particulier aux objets intellectuels, réaliste et substantialiste, qui seul a rendu possible le développement de la pensée critique et donc l'essor de la connaissance scientifique. Aussi, selon ce point de vue, les valeurs académiques liées à l'idée de " skholè » (de libre activité intellectuelle désintéressée) ne sont-elles pas les produits d'un préjugé intellectualiste dont il conviendrait de se débarrasser au plus vite en ouvrant l'école sur la «vie », mais un héritage précieux que toute tentative de réforme et d'amélioration des institutions éducatives se devrait de préserver.

S'il est quelque chose à reprocher aux pratiques pédagogiques traditionnelles, par conséquent, leur imperfection, selon Popper, ne réside pas là où la situe Dewey. Contrairement à ce qu'affirme ce dernier, l'autoritarisme de l'école classique, son caractère dogmatique et imparfaitement rationaliste, ne sont pas dus au privilège qu'elle accorde aux disciplines académiques ni à la clôture qu'elle institue par rapport à l'univers social - tous ces caractères étant la conséquence de la dimension «objective » (au sens poppérien du terme) des savoirs 
qu'elle transmet. II réside ailleurs, dans le fait que les façons ordinaires d'enseigner sont conçues pour exercer un pouvoir sur les croyances et les états mentaux du sujet. En raison de leurs présupposés épistémologiques subjectivistes, en effet, les pratiques usuelles d'enseignement assimilent à tort l'appropriation intellectuelle d'un savoir objectif par l'élève à un processus de modification de ses croyances: enseigner, selon ce point de vue, revient toujours à amener l'élève à croire quelque chose, à susciter de sa part une adhésion intime, la différence entre l'enseignement et la prédication (ou encore l'endoctrinement) ne résidant dès lors que dans l'obligation pour le maître de fournir à l'élève de «bonnes raisons » de croire (des preuves, des démonstrations). D'où il suit, selon Popper, que l'enseignement traditionnel présente le défaut de n'être pas radicalement affranchi de toute dimension religieuse et autoritaire, autrement dit de n'être que partiellement et imparfaitement laïque ${ }^{3}$. L'épistémologie poppérienne, on le voit, autorise une critique de la pédagogie classique d'une toute autre nature que celle de Dewey: une critique qu'on peut dire non progressiste de celle-ci (en ce qu'elle n'implique ni le rejet des valeurs académiques liées à l'idée classique de vérité ni donc la réduction de la connaissance à un processus d'interaction adaptative avec un environnement physique et social).

\section{- L'école et le religieux}

C'est peut-être par l'intention radicalement laïque qui vient d'être mise en évidence (laquelle est également soulignée par Alain Boyer, 1994), que la philosophie de Popper s'écarte le plus complètement de celle de Dewey. La pensée pédagogique du philosophe américain, en effet, débouche de façon tout à fait assumée et explicite sur une promotion de " l'attitude religieuse ", Dewey voyant dans la conscience de la communauté de destin révélée par l'entreprise scientifique la possibilité de donner un sens humaniste et immanent au sentiment religieux. Ainsi la critique de la religion (entendue comme croyance en un arrière monde transcendant) qui découle du progrès scientifique n'implique-t-elle nullement celle du "sentiment religieux » (Dewey, 2008, p.318), compris comme « sens des possibilités de l'existence et comme dévotion à la cause de ces possibilités, telle qu'on peut la distinguer de l'acceptation de ce qui est donné à une époque » (p.318). Selon Dewey, au contraire, la connaissance rationnelle et sa diffusion par l'éducation doivent répandre dans la société une «foi religieuse » qui serait "vouée à l'inspiration et à l'inculcation d'un sentiment des possibilités idéales dans le monde effectif » (p.325), laquelle accompagnerait, sans jamais s'y opposer, le progrès scientifique.

Une telle tentative pour concilier, moyennant la distinction entre la religion et le religieux, la pensée scientifique et l'attitude religieuse est inconcevable dans le cadre du "rationalisme critique » poppérien. Tel que l'entend Popper, en effet, l'attitude rationaliste n'impose pas seulement une rupture avec la foi en une entité transcendante, mais une rupture avec le régime de la croyance en général, avec le «religieux » non moins qu'avec la « religion » proprement dite. Si l'épistémologie poppérienne mérite le qualificatif de «laïque », c'est justement qu'elle vise à instituer une distinction absolument radicale - générique et non spécifique - entre le domaine de la croyance et celui de la rationalité, la connaissance rationnelle n'étant pas une certaine « espèce » de croyance (une croyance «fixée » car justifiée par de bonnes raisons de croire - preuves, démonstration, etc.) - mais tout autre chose qu'une croyance: une connaissance «objective ", "sans sujet connaissant ». Par où l'on voit qu'il n'est pas de place, au sein du rationalisme critique, pour l'exaltation d'un quelconque "sentiment religieux », fût-il distinct de toute adhésion à une « religion ».

Cette inspiration radicalement laïque de la pensée poppérienne est également manifeste dans le domaine politique et moral, où elle s'oppose, une fois encore, aux thèses soutenues par Dewey. Selon les analyses développées dans la Société ouverte et ses ennemis, en effet, les progrès du rationalisme, en matière d'organisation de la société, se marquent avant tout par le passage d'une conception concrète et affective du lien social, supposant une adhésion intime du sujet une « foi » commune, des valeurs et des idéaux « partagés " - à une conception juridique et

\footnotetext{
${ }^{3}$ Le terme «laïque ", qui n'a pas d'équivalent exact en anglais ni en allemand, ne se rencontre pas dans les écrits de Popper lui-même. Alain Boyer (1994, p.87) a cependant montré que ce qualificatif peut être légitimement appliqué à l'épistémologie poppérienne, en tant que celle-ci « se présente comme une tentative pour sortir sérieusement des modes de pensée religieux en philosophie ». C'est en ce sens que nous l'empruntons, dans l'analyse qui suit, à cet auteur.
} 
abstraite de celui-ci, exclusive de tout appel à une forme de croyance et de conviction collective. La démocratie, chez Popper, est avant tout un certain type d'organisation institutionnelle permettant le contrôle de l'action des gouvernants par les gouvernés et non un idéal social, imposant la diffusion d'une quelconque disposition commune parmi les citoyens. Tout autre, comme on sait, est le point de vue de Dewey pour qui la démocratie est d'abord une réalité sociale avant d'être une réalité politique et institutionnelle : la démocratie « est plus qu'une forme de gouvernement; elle est d'abord un mode de vie associé, d'expériences communes communiquées » (Dewey, 2011, p.169). Démocratie participative et coopérative d'un côté, démocratie institutionnelle et représentative de l'autre.

Les deux conceptions, bien évidemment, débouchent sur des visions différentes et opposées de la responsabilité politique et éthique de l'école. Chez Dewey l'institution scolaire se voit confier la tâche d'assurer la transmission des "dispositions affectives et intellectuelles semblables" (Dewey, 2011, p.83) nécessaires à la « vie communautaire », le but étant d'amener l'individu à "partager les intérêts, les objectifs et les idées communément admises par le groupe social » (p.89). Popper, de son côté, ne rejette pas l'idée que la transmission de la tradition rationaliste passe par la diffusion, à l'école, d'une certaine disposition affective, à savoir la " haine de la violence » (Popper, 1983, p.519) qui, seule, peut motiver le choix en faveur de la raison. Cette fonction " éducatrice » de l'école, toutefois, ne va pas au-delà. Parce que la démocratie, telle que la conçoit Popper, est un cadre juridique rassemblant des individus poursuivant chacun librement leurs fins propres - élevées ou basses, altruistes ou égoïstes peu importe - il ne peut entrer dans les finalités d'une éducation authentiquement libérale de répandre parmi les jeunes esprits une quelconque "foi commune ", quand bien même celle-ci serait exclusive de tout rapport à une entité transcendante.

\section{Conclusion}

Que conclure, au final, de cette rapide confrontation entre les pensées de Popper et de Dewey? À tout le moins qu'il est possible d'interroger les thèses du philosophe américain ainsi que les idées qu'elles ont inspirées sans défendre pour autant un retour à des conceptions épistémologiques ni à des pratiques pédagogiques traditionnelles. Autrement dit, qu'il est possible d'envisager une critique non conservatrice du progressisme contemporain. Le reproche fondamental que l'on peut adresser à la pensée de Dewey en se basant sur les analyses poppériennes, en effet, n'est pas de s'en être pris à l'épistémologie et à la pédagogie classiques, mais de l'avoir fait pour de mauvaises raisons, qui manquent la véritable nature du dogmatisme dont elles sont porteuses. Si l'on suit Popper, nous l'avons vu, le caractère dogmatique et autoritaire de l'éducation traditionnelle ne provient pas de son attachement aux valeurs intellectualistes issues de la philosophie grecque et de la notion classique de "skholè" (promotion de la connaissance "désintéressée ", supériorité des savoirs "théoriques » par rapport aux activités « pratiques », etc.) mais de ce qu'elle assigne à l'enseignement l'objectif de produire un effet psychologique sur l'élève, de susciter en lui une transformation mentale plus ou moins radicale (confondant ainsi le logique avec le psychologique, l'assimilation intellectuelle d'une connaissance objective avec un changement de croyances). Son dogmatisme provient, autrement dit, d'une incapacité à distinguer clairement la transmission des savoirs d'un processus de conversion de nature sinon religieuse du moins inauthentiquement laïque. Envisagé de ce point de vue, le modèle pédagogique proposé par Dewey ne constitue peut-être pas une rupture avec l'enseignement traditionnel aussi radicale et libératrice que le prétendent les partisans actuels du progressisme. N'est-il pas permis d'y voir le prolongement, et même à certains égards la confirmation et l'amplification, de cette philosophie de la croyance, d'inspiration secrètement religieuse, dont Popper estime que le rationalisme classique n'est jamais parvenu à s'affranchir totalement ? Les analyses qui précèdent, bien entendu, ne prétendent pas apporter des réponses à ces questions. Tout ce qu'elles se proposent d'établir est qu'on peut être conduit à interroger sous cet angle les idées de Dewey et des auteurs qui s'en sont inspirés en prenant pour guide une pensée, celle de Popper, qui n'est porteuse 
d'aucune intention conservatrice, ne vise la restauration d'aucun ordre ancien, épistémologique ou pédagogique.

\section{Références}

ARENDT Hannah (1972/1958), La crise de la culture, Paris, Gallimard.

BOYER Alain (1994), Introduction à la lecture de Karl Popper, Paris, Presses de l'ENS.

CHAUVIRÉ Christiane (2003), «Faillibilisme, hasard et logique de la découverte chez Peirce et Popper », dans Christiane Chauviré, Le grand miroir : essais sur Peirce et sur Wittgenstein, Besançon, Presses Universitaires Franc-Comtoises, p.123-138.

DEWEY John (2011), Démocratie et éducation suivi de Expérience et éducation, Paris, Colin.

- (2008), La quête de la certitude, Paris, Gallimard.

- (1993), Logique, La théorie de l'enquête, Paris, Presses Universitaires de France.

FABRE Michel (2016), «La faute à Dewey. À propos de quelques contresens sur sa philosophie de l'éducation », Recherches en éducation, $\mathrm{n}^{\circ} 28, \mathrm{p} .124-136$.

FOUREZ Gérard (2003), Apprivoiser l'épistémologie, Bruxelles, De Boeck.

GIORDAN André (2016), Apprendre !, Paris, Belin.

JOHSUA Samuel \& DUPIN Jean-Jacques (2003), Introduction à la didactique des sciences et des mathématiques, Paris, Presses Universitaires de France.

LE MOIGNE Jean-Louis (1999), Les épistémologies constructivistes, Paris, Presses Universitaires de France.

POPPER Karl (2006), Frühe Schriften, Gesammelte Werke in deutscher Sprache, I, Tübingen, Mohr Siebeck.

- (1997), Toute vie est résolution de problèmes, Paris, Acte Sud.

- (1991), La connaissance objective, Paris, Flammarion.

- (1990), Le réalisme et la science, post-scriptum à La Logique de la découverte scientifique, Paris, Hermann.

- (1989), Le mythe du cadre de référence, dans R. Bouveresse (éd.), Karl Popper et la science d'aujourd'hui, Paris, Aubier.

- (1985), Conjectures et réfutations, Paris, Payot. 